\title{
Regulation of cytochrome P450 3A4 by small vault RNAb derived from the non-coding vault RNA1 of multidrug resistance-linked vault particle
}

\author{
CHUNJIE MENG ${ }^{1}$, ZHIYUN WEI $^{1,2}$, YITING ZHANG ${ }^{1}$, LIANG YAN $^{3}$, \\ HANG $\mathrm{HE}^{3}$, LIRONG ZHANG ${ }^{3}$ and QINGHE XING ${ }^{1}$ \\ ${ }^{1}$ Children's Hospital and Institutes of Biomedical Sciences, Fudan University, Shanghai 200032, P.R. China; \\ ${ }^{2}$ Department of Neurology, Brigham and Women's Hospital, Harvard Medical School, Boston, MA 02115, USA; \\ ${ }^{3}$ Department of Pharmacology, School of Medicine, Zhengzhou University, Zhengzhou, Henan 450006, P.R. China
}

Received May 8, 2015; Accepted January 25, 2016

DOI: $10.3892 / \mathrm{mmr} .2016 .5228$

\begin{abstract}
Cytochrome P450 3A4 (CYP3A4) is the most abundant cytochrome P450 enzyme in human liver and intestine, contributing to the metabolism of $>60 \%$ of all pharmaceuticals. The expression levels of hepatic CYP3A4 show great inter-individual variation. However, the detailed regulatory mechanism of CYP3A4 expression has remained largely elusive. It has been reported that the non-coding RNA small vault (sv)RNAb targets the 3' untranslated region (3'UTR) of CYP3A4 in MCF7 cells. However, to date, the role of svRNAb has not been examined in human liver tissue and hepatic cell lines such as HepG2, which was the aim of the present study. Polymerase chain reaction analysis indicated that the expression of CYP3A4 was significantly different within a study cohort $(n=19)$. In addition, a significant negative correlation was observed between svRNAb and CYP3A4 expression in human liver tissue samples. Furthermore, a luciferase assay on HepG2 cells verified that svRNAb directly targets CYP3A4 and regulates the expression of CYP3A4 by interacting with the validated binding sites of the CYP3A4 3'UTR. The results provided insight into the variation of the expression of CYP3A4 among individuals and provided a novel method for the adjustment of personalized drug treatment. Furthermore, the present study provided a mechanism of the regulatory role of svRNAb in multidrug-resistant cells.
\end{abstract}

Correspondence to: Professor Qinghe Xing, Children's Hospital and Institutes of Biomedical Sciences, Fudan University, 138 Yixueyuan Road, Shanghai 200032, P.R. China

E-mail: xingqinghe@hotmail.com

Professor Lirong Zhang, Department of Pharmacology, School of Medicine, Zhengzhou University, 100 Kexue Road, Zhengzhou, Henan 450006, P.R. China

E-mail: lrzhang@zzu.edu.cn

Key words: cytochrome P450 3A4, small vault RNAb, gene regulation, multidrug resistance

\section{Introduction}

Cytochrome P450 (CYP)3A4 is the most abundant hepatic and intestinal CYP450 enzyme in humans, contributing to the metabolism of most drugs (1). It shows large intra- and inter-individual variations, which contribute to marked individual differences in drug responses in terms of therapeutic effects as well as adverse effects (2). High CYP3A4 activity accelerates the metabolic rate of drugs, shortens their half-life and reduces their plasma concentration. Therefore, in individuals with high enzyme activity, the anticipated therapeutic efficacy of certain drugs may not be achieved at conventional doses. By contrast, individuals with low CYP3A4 activity may show decreased drug clearance as well as prolonged half-life and obvious accumulation of drugs. Collectively, the variability of CYP3A4 activity or expression may lead to the uncertainty of the therapeutic efficacy and studies on the inter-individual variability of CYP3A4 may facilitate the process of individualized pharmacotherapy and reduce adverse effects of drugs (1,3).

Inter-individual variability in CYP3A4 expression is thought to be largely heritable $(4,5)$, and $>30$ single nucleotide polymorphisms have been identified in the CYP3A4 gene. However, the currently known genetic variants at the CYP3A4 locus are unlikely to account for the proposed high variability in CYP3A4-associated metabolic function (4,6-9). CYP3A4 can be induced by a variety of structurally diverse xenochemicals via xenobiotic receptors (10-19). For instance, grapefruit juice is a potent inhibitor of CYP3A4-mediated drug metabolism (20). Under specific pathophysiological conditions, such as inflammatory stimulation, CYP3A4 can also be induced, which is a key process involved in the toxic vs. therapeutic effects of numerous drugs $(21,22)$. Furthermore, it has been shown that CYP3A4 activity is higher in women than in men, suggesting enhanced pharmacokinetics in women (23). In spite of this knowledge, the detailed underlying mechanisms of the variation of CYP3A4 expression among individuals has remained elusive.

Vault particles are intracellular ribonucleoprotein particles containing three different proteins and non-coding 
vault RNAs (vRNAs), termed as vRNA1, vRNA2 and vRNA3 (24-26), and are thought to be implicated in multidrug resistance $(10,25,27-29)$. Studies on the biological roles of vaults have focused on their protein components, while vRNAs have not been functionally characterized. As vRNAs can theoretically fold into structures resembling micro (mi)RNA precursors, Persson et al (30) sequenced a group of $\sim 23$ nucleotide-containing small RNAs matching the RNA components of the vault particle and discovered the non-coding RNA svRNAb.

svRNAb, a $\sim 23$ nucleotide non-coding RNA, is encoded by the 5 '-arm of the stem-loop structure of non-coding vault RNA1 (vRNA1). pre-svRNAb, a 32 nucleotide containing RNAs derived from vRNA1 and vRNA2, ends precisely at the 3 ' terminus of svRNAb. svRNAb can associate with Argonaute proteins to guide sequence-specific cleavage and regulate gene expression in the pattern similar to that of miRNAs. CYP3A4 has been validated as the target gene of svRNAb in the MCF7 cell line (30).

In the light of the importance of CYP3A4 in the liver, the present study was the first to investigate the role of svRNAb in the regulation of CYP3A4 expression in liver tissue samples and the HepG2 cell line. The present study also quantified svRNAb by reverse transcription-quantitative polymerase chain reaction (RT-qPCR). The results revealed a significant correlation between CYP3A4 and svRNAb in human liver tissue samples. Furthermore, a luciferase assay validated that svRNAb directly targets the 3'-untranslated region (3'-UTR) of CYP3A4 in the HepG2 cell line. Furthermore, the present study suggested a possible role for svRNAb in drug metabolism via regulation of CYP3A4 expression in multidrug-resistant cells.

\section{Materials and methods}

Sample collection. Human liver tissue samples were obtained from 19 Han Chinese donors who underwent surgery at the First Affiliated Hospital of Zhengzhou University (Zhengzhou, China) with the donors' written informed consent. The present study was approved by the Clinical Research Ethics Committee of the First Affiliated Hospital of Zhengzhou University and complied with the Declaration of Helsinki and its subsequent revisions. A total of 14 males and 5 females (age, 33-60 years; mean age, 50.3 years) were included in the study. Among these donors, eight had mild cirrhosis and none had Hepatitis B or C. All donors had normal liver functions. To avoid any inhibitory or stimulatory effects on CYP3A4 activity, none of the donors received any pre-operative medication affecting CYP3A4, such as rifampicin, dexamethasone or propofol for two weeks, or alcohol, grapefruit juice or caffeine within three days prior to the surgery.

CYP3A4 enzymatic activity. Liver microsomes were extracted from tissues using the $\mathrm{CaCl}_{2}$ (Sigma-Aldrich, St. Louis, MO, USA) method. Liver microsomes were incubated with midazolam $\left(0.25-75 \mathrm{mmol} / \mathrm{l}\right.$; Sigma-Aldrich) at $37^{\circ} \mathrm{C}$ for $10 \mathrm{~min}$. The 1-OH midazolam concentration was measured using an Odyssil C18 HPLC system (Agela Technologies, Wilmington, DE, USA) with a mobile phase of $20 \mathrm{mM} / 1$ ammonium acetate-acetonitrile, a column temperature of $40^{\circ} \mathrm{C}$ and a flow rate of $1 \mathrm{ml} / \mathrm{min}$. Michaelis constants $(\mathrm{Km}$,
Table I. Population variability of hepatic CYP3A4 expression phenotypes $(n=19)$.

\begin{tabular}{lcc}
\hline Parameter & $\begin{array}{c}\text { CYP3A4/GAPDH mRNA } \\
\text { Relative units }\end{array}$ & $\begin{array}{c}\text { Enzyme } \\
\text { activity }\end{array}$ \\
\hline Median & 0.96 & 8.36 \\
Minimum & 0.06 & 1.78 \\
Maximum & 3.45 & 30.17 \\
Ratio max/min & 55.18 & 16.91 \\
Normal distribution & No & No \\
Coefficient of & 89.05 & 69.61 \\
variation (\%) & & \\
\hline
\end{tabular}

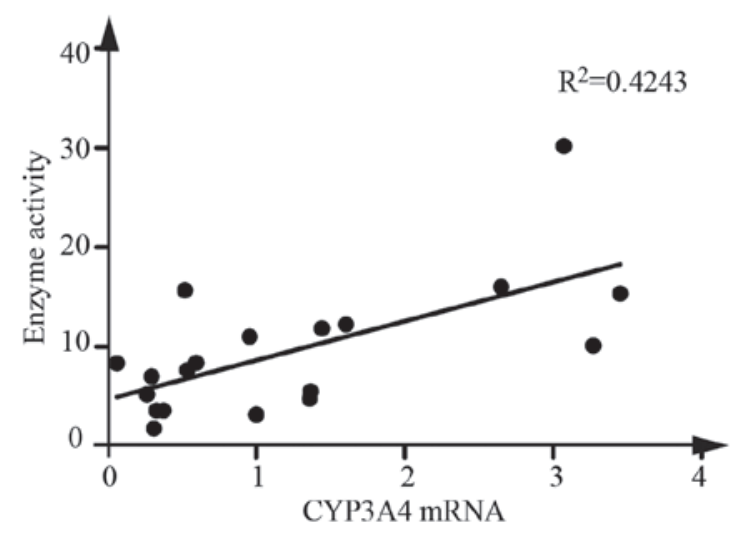

Figure 1. Spearman correlation of hepatic CYP3A4 mRNA and enzyme activity. CYP3A4 mRNA and enzyme activity were significantly correlated.

$\mathrm{mM})$ were estimated to quantify enzymatic activity with five replicates (31).

RT-qPCR analysis. Approximately $0.1 \mathrm{~g}$ of liver was cut into small pieces and ground with a grinding rod (Shanghai Ding Jie Technology, Ltd., Shanghai, China) while total RNA was extracted using $1 \mathrm{ml}$ TRIzol (Life Technologies; Thermo Fisher Scientific, Inc., Waltham, MA, USA) according to the manufacturer's instructions, followed by gel electrophoresis for quality control. Total RNAs were reverse-transcribed to cDNA using a Revert Aid First Strand cDNA Synthesis kit (Thermo Fisher Scientific, Inc.). cDNA was then amplified by PCR using FastStart Universal SYBR Green Master Mix (Roche, Basel, Switzerland) with the following primers (Invitrogen; Thermo Fisher Scientific, Inc.): CYP3A4 forward, 5'-CCA AGCTATGCTCTTCACCG-3' and reverse, 5'-TCAGGCTCC ACTTACGGTGC-3'; GAPDH forward, 5'-ATCACCATC TTCCAGGAGCGA-3' and reverse, 5'-GCTTCACCACCT TCTTGATGT-3') The thermocycling conditions included 1 cycle at $95^{\circ} \mathrm{C}$ for $10 \mathrm{~min}$, and 40 cycles of $95^{\circ} \mathrm{C}$ for $15 \mathrm{sec}$ and $60^{\circ} \mathrm{C}$ for $1 \mathrm{~min}$. Relative quantities were normalized to GAPDH as the endogenous control and were calculated using the $2^{-\Delta \Delta \mathrm{Cq}}$ method (32). For svRNAb quantification, RT-qPCR was performed using TaqMan ${ }^{\circledR}$ Small RNA Assays (Applied Biosystems; Thermo Fisher Scientific, Inc.). U6 small nuclear RNA (Invitrogen; Thermo Fisher Scientific, Inc.) was used as the endogenous control. The cycling conditions included 
A

SVRNAb 3'GACAGCUUCAUUGGCGACUCGAU 5'

pre-svRNAb 3'GACAGCUUCAUUGGCGACUCGAUUUCGGUCGG 5'

B
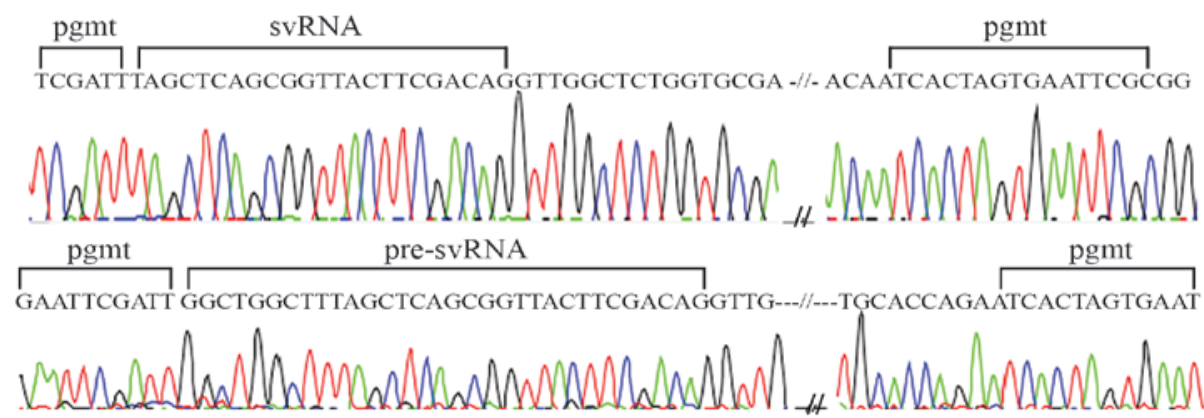

C

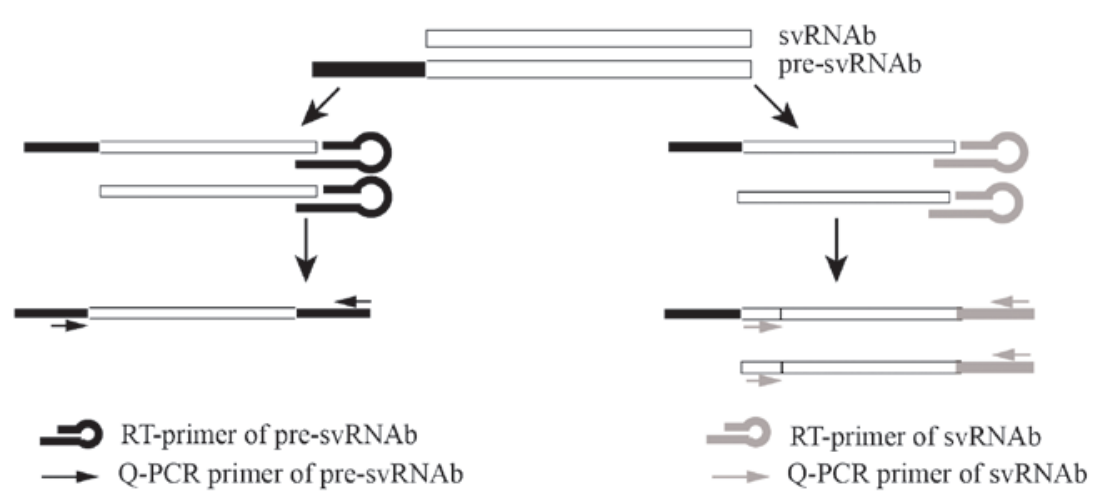

Figure 2. Sequencing results and the Q-PCR process of svRNAb and pre-svRNAb. (A) Sequence analysis showed that pre-svRNAb ends precisely at the 3 terminus of svRNAb. (B) The sequencing results showed that the primers for svRNAb and pre-svRNAb putatively amplified the specific products. (C) svRNAb and pre-svRNAb have identical 3' ends. In RT reactions, the stem loop RT primer can reversely transcribe the pre-svRNAb and svRNAb simultaneously. When svRNAb was quantified, pre-svRNAb was quantified at the same time. svRNA, small vault RNA; RT, reverse transcription; Q-PCR, quantitative polymerase chain reaction.

1 cycle at $95^{\circ} \mathrm{C}$ for $10 \mathrm{~min}$, and 40 cycles of $95^{\circ} \mathrm{C}$ for $15 \mathrm{sec}$ and $60^{\circ} \mathrm{C}$ for $1 \mathrm{~min}$. All qPCRs were run on a $7900 \mathrm{HT}$ instrument (Applied Biosystems) in three replicates.

Plasmid construction and sequencing. The target PCR products were recovered and ligated into the $\mathrm{pGMT}$ vector (Tiangen Biotech Co., Beijing, China). Next, PCR products were further separated using electrophoresis on $2 \%$ agarose gels (Tiangen Biotech Co.). A Zymoclean ${ }^{\mathrm{TM}}$ Gel DNA Recovery kit (Zymo Research Corp., Irvine, CA, USA) was used to recover pure DNA from agarose gels. Through TA cloning with EcoV (New England BioLabs, Inc., Ipswich, MA, USA), the PCR products of svRNAb and pre-svRNAb were inserted into the PGMT vector. The universal forward primer (Invitrogen; Thermo Fisher Scientific, Inc.; 3'-TGTAATACGACTCACTAT AGGG-5') was used for sequencing, which was conducted by Major BioShanghai Technologies Co., Ltd. (Shanghai, China).

Dual-luciferase assay. HepG2 cells (Fudan University IBS Cell Bank, Shanghai, China) were cultured in Dulbecco's modified Eagle's medium (Invitrogen; Thermo Fisher Scientific, Inc.) containing $10 \%$ fetal bovine serum (Invitrogen; Thermo Fisher Scientific, Inc.) at $37^{\circ} \mathrm{C}$ in a humidified atmosphere of $5 \% \mathrm{CO}_{2}$. For validation of CYP3A4 as a direct target of svRNAb, a luciferase reporter assay was performed using the pGL3 Luciferase Reporter Vector containing wild-type (WT) CYP3A4 3'-UTR and mutant (MT) CYP3A4 3'-UTR. Total RNA was reverse transcribed using a RevertAid First Strand cDNA Synthesis kit (Thermo Fisher Scientific, Inc.) according to the manufacturer's instructions. A 931 base-pair fragment of the CYP3A4 3'-UTR covering all three svRNAb seed matches was amplified from cDNA of the human liver tissue using specific primers obtained from Invitrogen (Thermo Fisher Scientific, Inc.; forward, 5'-GAATTCTCTAGATGTGCC TGAGAACACCAGAG-3' and reverse, 5'-GAATTCTCT AGAACGTGCTTCAAAAAGGCATA-3') which have XbaI sites. The PCR products were purified using DNA Clean \& Concentrator $^{\mathrm{TM}}-5$ (Zymo Research Corp.) according to the manufacturer's instructions. Following the XbaI enzyme digestion (New England BioLabs, Inc.) of the purified product and the pGL3 Renilla luciferase vector (Promega Corp. Madison, WI, USA), the plasmids were ligated with T4 DNA ligase (New England BioLabs, Inc.). Deletions of seed matches were sequentially introduced using the KOD-Plus-Mutagenesis kit (Toyobo Co. Ltd., Osaka, Japan). The following primers (Invitrogen; Thermo Fisher Scientific, Inc.) were used: Forward, 5'-ATGCATGTACAGAATCCC CGGTTA-3' and reverse, 5'-CTCTCATTGTCTGTGTAG AGTGTTATAC-3' (site 1); forward, 5'-GAGGAGTTAATG GTGCTAACTGG-3' and reverse, 5'-CTGATAAGAGAA TCAACATTTCTCAATAAT-3' (site 2); and forward, 5'-TTC AACATCCGCCTCCCAGGTT-3' and reverse 5'-TGAGAT TGCACCACTGCACTCC-3' (site 3). Co-transfection experi- 
A

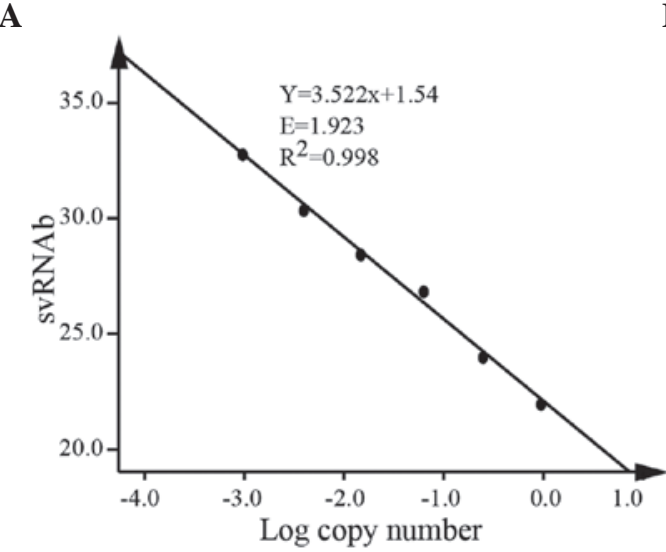

C

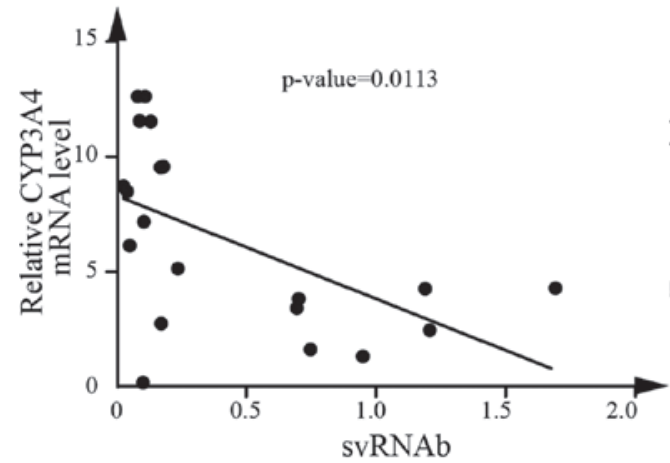

B

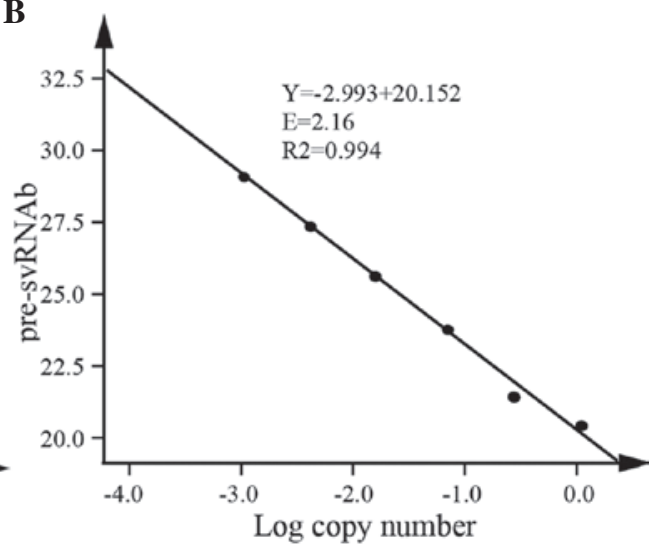

D

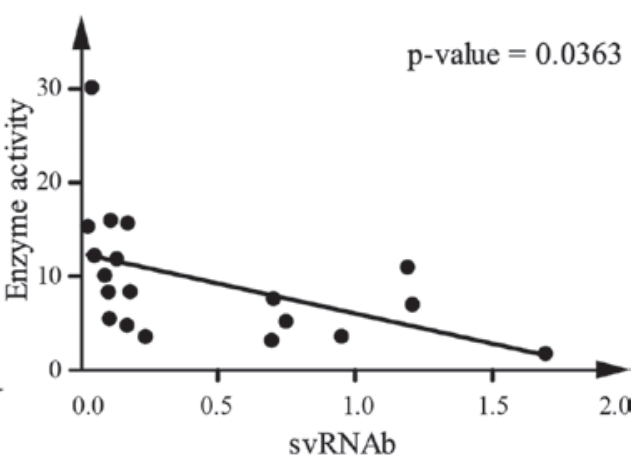

$\mathbf{E}$

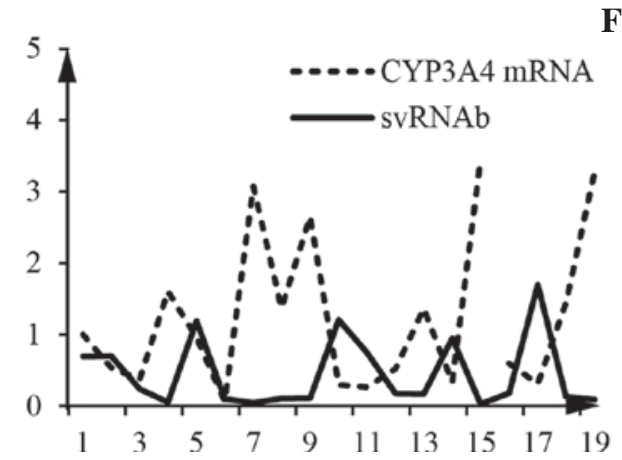

F

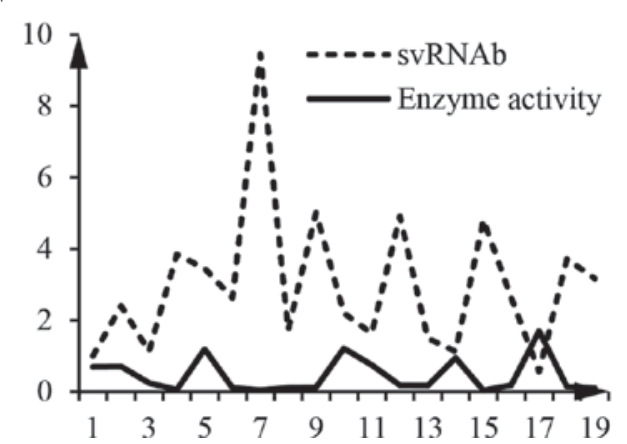

Figure 3. Establishment of methods of quantifying the svRNAb and Spearman correlations analysis of CYP3A4 mRNA and svRNAb. (A and B) Polymerase chain reaction amplification efficiency of primer pair svRNAb and pre-svRNAb. The efficiency was 1.92 and 2.16 for svRNAb and pre-svRNAb, respectively. (C) As determined by Spearman correlation analysis, there was significant correlation between CYP3A4 mRNA levels and svRNAb levels. (D) svRNAb was negatively correlated with CYP3A4 mRNA. In individuals within the study cohort $(n=19)$, (E) a negative correlation between the expression of svRNAb and CYP3A4 mRNA as well as (F) a negative correlation between the expression of svRNAb and CYP3A4 enzyme activity was observed. svRNA, small vault RNA; E, efficiency; CYP, cytochrome P.

ments were performed in 96-well plates. A total of $100 \mathrm{ng}$ WT or MT reporter constructs and $50 \mathrm{nM}$ svRNAb mimics or negative control mimics (JIMA, Shanghai, China) were co-transfected into HepG2 cells using Lipofectamine 2000 transfection reagent (Invitrogen) according to the manufacturer's instructions. After $48 \mathrm{~h}$, luciferase activity was measured with the dual luciferase reporter assay system (Promega Corp.). The relative luciferase activity was normalized to that of firefly luciferase.

Statistical analysis. Each experiment was performed at least three times. All values are expressed as the mean \pm standard deviation. The establishment of the standard curve of svRNAb and Spearman's rank correlation were performed using SPSS for Windows, version 11.0 (International Business Machines,
Inc., Armonk, NY, USA). All tests were two-tailed and $\mathrm{P}<0.05$ was considered to indicate a statistically significant difference.

\section{Results}

Inter-individual variability of hepatic CYP3A4. CYP3A4 varied considerably at the mRNA expression level as well as in terms of enzyme activity in the 19 liver tissue samples assessed in the present study (Table I). The variation was 55-fold and 17-fold for mRNA and enzyme activity, respectively. The coefficient of variation as a normalized measure of variability was then calculated to be 89.05 for mRNA expression and 69.61 for enzyme activity. These values are comparable to those of a previous study on CYP3A4 
A

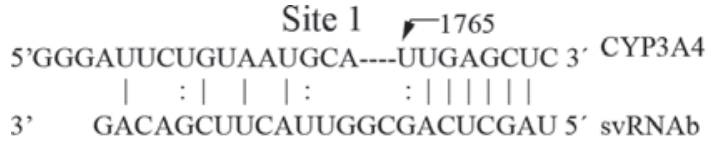

Site $2 \curvearrowleft 1906$

5'GCACCAUUAACUCCUC-CUGAGCUC 3' CYP3A4

$:|||:||||||| \mid$

3' GACAGCUUCAUUGGCGACUCGAU 5' svRNAb

\begin{tabular}{|c|c|}
\hline Site 3 & \\
\hline $\begin{array}{l}\text { 5'GAGGCGGAUGUUGAAGUGAGCUGA 3' } \\
\qquad|||:||||| \mid: \text { : }\end{array}$ & CYP3A4 \\
\hline ACAGCUUCAUUGGCGACUCGAU $5^{\prime}$ & RNAb \\
\hline
\end{tabular}

B

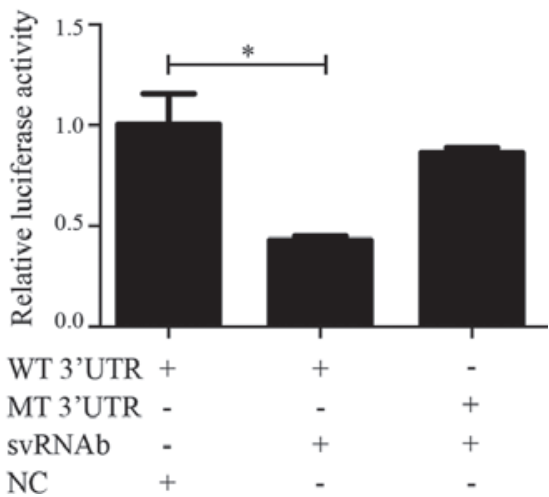

Figure 4. The 3'UTR of CYP3A4 mRNA is targeted by svRNAb-mimics in the HepG2 cell line. (A) Predicted seed matches for svRNAb in the CYP3A4 3'UTR with nucleotide positions in Ensembl ID ENSG00000160868 (http://grch37.ensembl.org/Homo_sapiens/Gene/Summary?db=core;g=ENSG00000160 868;r=7:99354604-99381888). (B) Relative luciferase activity in groups transfected with different factors. Values are expressed as the mean \pm standard deviation. "P<0.05 vs. the CYP3A4 WT + svRNAb-NC and CYP3A4 MT + svRNAb groups. WT, wild-type; MT, mutant; NC, negative scrambled control; UTR, untranslated region; CYP cytochrome P; svRNA, small vault RNA.

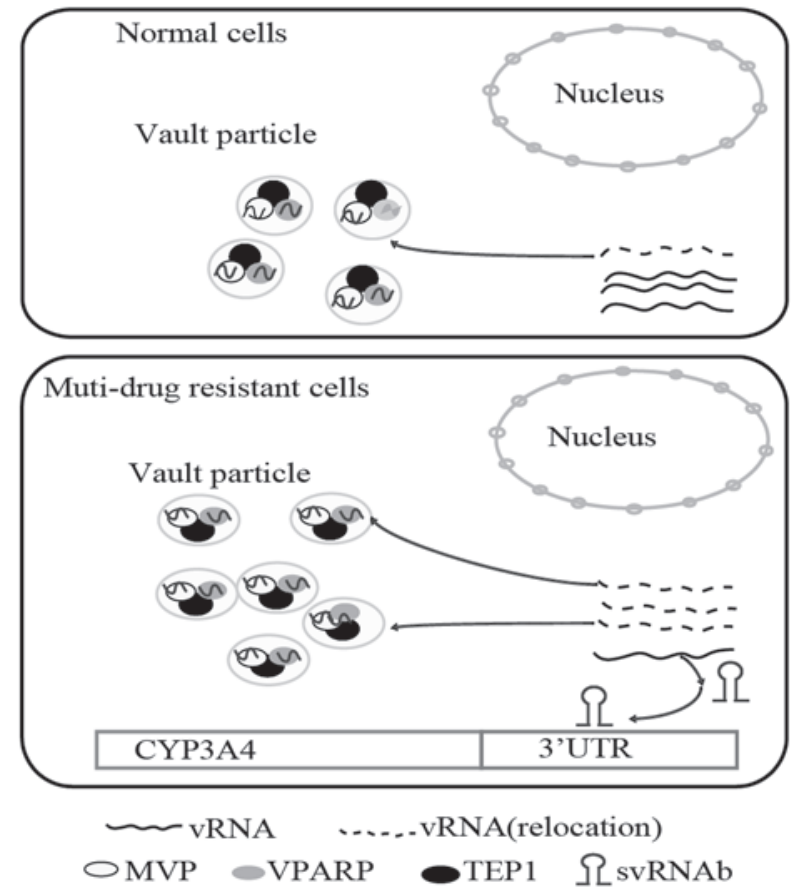

Figure 5. Schematic illustrating the putative role of svRNAb in multi-drug resistant cells. vRNAs are located to vault particles as well as freely abundant in the cytoplasm. In multidrug-resistant cells, vRNAs relocate from the cytoplasm and form excess vault particles. Consequently, svRNAb is reduced in the cytoplasm and the expression of CYP3A4 increases. Concomitantly, the first pass effect the liver is enhanced, and the efficacy of certain drugs is weakened. MVP, major vault protein; vPARP, vault poly-(adenosine diphosphate ribose) polymerase; TEP1, telomerase-associated protein; svRNA, small vault RNA.

variation (7). Furthermore, a Spearman correlation analysis revealed that CYP3A4 mRNA expression was significantly correlated with the enzyme activity (Fig. 1).

PCR amplification of $s v R N A b$ and pre-svRNAb. As shown in Fig. 2A, pre-svRNAb has an identical $3^{\prime}$ end to that of svRNAb, while the former is nucleotides longer at the $5^{\prime}$ end. As the abundance of svRNAb was expected to be low in liver tissues, the present study tested whether the PCR primers for svRNAb and pre-svRNAb were able to produce specific amplification products. Direct sequencing of amplification products confirmed that the PCR primers efficiently amplified the specific products (Fig. 2B). Due to the partial structural identify of pre-svRNAb and svRNAb, the stem loop RT primer can reversely transcribe pre-svRNAb as well as svRNAb in the RT reactions. The PCR primer for pre-svRNAb amplified pre-svRNAb only, while the PCR primer for svRNAb amplified svRNAb and pre-svRNAb (Fig. 2C).

CYP3A4 is correlated with svRNAb in liver tissue samples. For accurate quantification, qPCR was used to quantify pre-svRNAb and the sum of svRNAb and pre-svRNAb, with the primer sets designed for pre-svRNAb and svRNAb, respectively. First, standard curves were generated. The amplification efficiency of svRNAb was 1.92 and that of the pre-svRNAb was 2.16 (Fig. 3A and B). The calculated difference of copy numbers between these two sets of primers was equal to the copy number of svRNAb. Using this method, the expression levels of svRNAb in 19 human liver tissue samples were measured. The results revealed a statistically significant correlation between CYP3A4 mRNA and svRNAb (Fig. 3C). Enzyme activity of CYP3A4 was also negatively correlated with the svRNAb (Spearman, P<0.05) (Fig. 3D). Analysis of 19 liver tissue samples implied that svRNAb had a negative correlation with CYP3A4 mRNA and enzyme activity, respectively (Fig. 3E and F). All of these results indicated that svRNAb regulates CYP3A4 expression and contributes to the variability of the expression of CYP3A4.

svRNAb directly regulates CYP3A4 expression in Hep G2 cells. Three binding sites for svRNAb are present in the 3'-UTR of the CYP3A4 transcript (30) (Fig. 4A). To identify whether svRNAb targeted CYP3A4 in HepG2 cells via these three binding sites, a dual-luciferase reporter gene assay was performed. Luciferase reporter plasmids containing the wild-type 3'-UTR (Luc-CYP3A4-wt) or mutant 3'-UTR (Luc-CYP3A4-mt) of CYP3A4 were constructed to verify the targeted region. The three validated binding sites in the 3 '-UTR were mutated simultaneously and every corresponding svRNAb binding site 
had one deletion mutation. The results showed that svRNAb significantly decreased the firefly luciferase activity of the reporter vector containing the wild-type $3^{\prime} \mathrm{UTR}(\mathrm{P}<0.05)$; however, the activity of the mutant 3 '-UTR vector remained unaffected $(\mathrm{P}>0.05)$ (Fig. 4B). These results indicated that svRNAb targeted the CYP3A4 gene through interacting with the three binding sites in its 3'-UTR in HepG2 cells, which was consistent with the result of a previous study (30). However, the expression of CYP3A4 in HepG2 cells was so low that the mRNA and protein was almost undetectable. Further study is also required to validate the expression of mRNA and protein after the transfection of svRNAb mimics into human primary hepatocytes.

svRNAb may be involved in drug metabolism by regulating CYP3A4 expression in multi-drug resistant cells. Vilalta et al (24) reported a dynamic association between the vRNA and vault particles, and that there was a pool of vRNAs, from which a certain fraction was re-located to constitute the vault particle at any given time (30). The present study demonstrated that in multi-drug resistant cells, vRNAs were shown to re-locate from the cytoplasm and form excess vault particles. vRNAs were located in vault particles as well as being freely abundant in the cytoplasm. As svRNAb was processed from vRNA1, it was reasonable to assume that excess formation of vault particles in multi-drug resistant cell lines would decrease the amount of free vRNA1 in the cytoplasm $(10,26)$. Thus, the present study speculated that svRNAb expression was reduced, while CYP3A4 mRNA was augmented in multi-drug resistant cell lines. The suggested mechanism is illustrated in the scheme shown in Fig. 5. From this viewpoint, is may be assumed that svRNAb is involved in drug metabolic mechanisms by regulating CYP3A4 expression in drug-resistant cells.

\section{Discussion}

CYP3A4 is the most abundant hepatic CYP450 enzyme in humans, contributing to the metabolism of most drugs in current clinical use. However, the detailed underlying mechanisms of inter-individual variability with regard to CYP3A4 levels and the resulting drug responses and metabolism have largely remained elusive. svRNAb, a newly identified non-coding RNA, has been found to target the CYP3A4 gene in the MCF7 cell line (30). The present study validated the association between svRNAb and CYP3A4 in human liver tissue samples and the HepG2 cell line.

A significant negative correlation was observed between svRNAb and CYP3A4 expression in human liver tissue samples. This hypothesis was further confirmed by a luciferase activity assay, which demonstrated that svRNAb was able to target the 3'UTR of CYP3A4, and the binding site was consistent with the seed regions previously reported (30). These data demonstrated that svRNAb is able to regulate CYP3A4 expression in the liver, which provided insight into the underlying mechanisms of the inter-individual variability of hepatic CYP3A4 expression.

It is worth pointing out a few limitations and drawbacks of the present study. Although Persson et al (30) detected the expression of svRNAb using RNase protection assays, the expression of svRNA in liver tissues remained undetectable. Furthermore, the PCR primer for svRNAb allowed for distinguishing svRNAb from pre-svRNAb. The present study was the first to quantitatively detect svRNAb expression using RT-qPCR. However, whether this method can quantify svRNAb accurately and effectively requires further evaluation. In addition, the mechanism provided by Persson et al (30) and the present study, suggesting that svRNAb is involved in drug metabolism by regulating CYP3A4 expression in multi-drug resistant cells, requires additional verification.

In conclusion, the present study reported that svRNAb is able to regulate the CYP3A4 expression in the liver, thereby providing insight into the mechanisms of inter-individual variability in the therapeutic and toxic effects of drugs.

\section{Acknowledgements}

The authors are grateful to all of the participants of this study. This work was supported by grants from the Shanghai Municipal Commission of Science and Technology Program (grant no. 14DJ1400100), the National Natural Science Foundation of China (grant nos. 30971582, 81261120400, 81173127 and 31371274) and the 973 Program (no. 2011CB504501).

\section{References}

1. Westlind A, Malmebo S, Johansson I, Otter C, Andersson TB, Ingelman-Sundberg $\mathrm{M}$ and Oscarson $\mathrm{M}$ : Cloning and tissue distribution of a novel human cytochrome p450 of the CYP3A subfamily, CYP3A43. Biochem Biophys Res Commun 281: 1349-1355, 2001.

2. Sevrioukova IF and Poulos TL: Understanding the mechanism of cytochrome P450 3A4: recent advances and remaining problems. Dalton transactions 42: 3116-3126, 2013.

3. de Wildt SN, Kearns GL, Leeder JS and van den Anker JN: Cytochrome P450 3A: Ontogeny and drug disposition. Clin Pharmacokinet 37: 485-505, 1999.

4. Lamba JK, Lin YS, Schuetz EG and Thummel KE: Genetic contribution to variable human CYP3A-mediated metabolism. Adv Drug Deliv Rev 54: 1271-1294, 2002.

5. Urquhart BL, Tirona RG and Kim RB: Nuclear receptors and the regulation of drug-metabolizing enzymes and drug transporters: Implications for interindividual variability in response to drugs. J Clin Pharmacol 47: 566-578, 2007.

6. Keshava C, McCanlies EC and Weston A: CYP3A4 polymorphisms-potential risk factors for breast and prostate cancer: A HuGE review. Am J Epidemiol 160: 825-841, 2004.

7. Wojnowski L and Kamdem LK: Clinical implications of CYP3A polymorphisms. Expert Opin Drug Metab Toxicol 2: 171-182, 2006.

8. Perera MA: The missing linkage: What pharmacogenetic associations are left to find in CYP3A? Expert Opin Drug Metab Toxicol 6: 17-28, 2010.

9. Klein K and Zanger UM: Pharmacogenomics of Cytochrome P450 3A4: Recent progress toward the 'Missing Heritability' problem. Front Genet 4: 12, 2013.

10. Kitazono M, Sumizawa T, Takebayashi Y, Chen ZS, Furukawa T, Nagayama S, Tani A, Takao S, Aikou T and Akiyama S: Multidrug resistance and the lung resistance-related protein in human colon carcinoma SW-620 cells. J Natl Cancer Inst 91: 1647-1653, 1999.

11. Willson TM and Kliewer SA: PXR, CAR and drug metabolism. Nat Rev Drug Discov 1: 259-266, 2002.

12. Timsit YE and Negishi M: CAR and PXR: The xenobiotic-sensing receptors. Steroids 72: 231-246, 2007.

13. Klein K, Thomas M, Winter S, Nussler AK, Niemi M, Schwab M and Zanger UM: PPARA: A novel genetic determinant of CYP3A4 in vitro and in vivo. Clin Pharmacol Ther 91: 1044-1052, 2012.

14. Oda Y, Nakajima M, Tsuneyama K, Takamiya M, Aoki Y, Fukami $\mathrm{T}$ and Yokoi T: Retinoid $\mathrm{X}$ receptor $\alpha$ in human liver is regulated by miR-34a. Biochem Pharmacol 90: 179-187, 2014. 
15. Yang J, Hao C, Yang D, Shi D, Song X, Luan X, Hu G and Yan B Pregnane $X$ receptor is required for interleukin-6-mediated down-regulation of cytochrome P450 3A4 in human hepatocytes. Toxicol Lett 197: 219-226, 2010.

16. Watanabe K, Sakurai K, Tsuchiya Y, Yamazoe Y and Yoshinari K: Dual roles of nuclear receptor liver X receptor $\alpha(\mathrm{LXR} \alpha)$ in the CYP3A4 expression in human hepatocytes as a positive and negative regulator. Biochem Pharmacol 86 : 428-436, 2013.

17. Goodwin B, Hodgson E, D'Costa DJ, Robertson GR and Liddle C: Transcriptional regulation of the human CYP3A4 gene by the constitutive androstane receptor. Mol Pharmacol 62: 359-365, 2002.

18. Wang K, Chen S, Xie W and Wan YJ: Retinoids induce cytochrome P450 3A4 through RXR/VDR-mediated pathway. Biochem Pharmacol 75: 2204-2213, 2008.

19. Thirumaran RK, Lamba JK, Kim RB, Urquhart BL, Gregor JC Chande N, Fan Y, Qi A, Cheng C, Thummel KE, et al: Intestinal CYP3A4 and midazolam disposition in vivo associate with VDR polymorphisms and show seasonal variation. Biochem Pharmacol 84: 104-112, 2012.

20. Jones SA, Moore LB, Shenk JL, Wisely GB, Hamilton GA, McKee DD, Tomkinson NC, LeCluyse EL, Lambert MH, Willson TM, et al: The pregnane $\mathrm{X}$ receptor: A promiscuous xenobiotic receptor that has diverged during evolution. Mol Endocrinol 14: 27-39, 2000

21. Aitken AE and Morgan ET: Gene-specific effects of inflammatory cytokines on cytochrome p450 2c, 2 b6 and 3a4 mrna levels in human hepatocytes. Drug Metab Dispos 35: 1687-1693, 2007.

22. Dickmann LJ, Patel SK, Wienkers LC and Slatter JG: Effects of Interleukin $1 \beta$ (IL-1 $\beta$ ) and IL-1 $\beta$ /interleukin 6 (IL-6) combinations on drug metabolizing enzymes in human hepatocyte culture. Curr Drug Metab 13: 930-937, 2012.
23. Wolbold R, Klein K, Burk O, Nüssler AK, Neuhaus P,Eichelbaum M, Schwab M and Zanger UM: Sex is a major determinant of CYP3A4 expression in human liver. Hepatology 38: 978-988, 2003.

24. Vilalta A, Kickhoefer VA, Rome LH and Johnson DL: The rat vault RNA gene contains a unique RNA polymerase III promoter composed of both external and internal elements that function synergistically. J Biol Chem 269: 29752-29759, 1994.

25. Kickhoefer VA, Rajavel KS, Scheffer GL, Dalton WS, Scheper RJ and Rome LH: Vaults are up-regulated in multidrug-resistant cancer cell lines. J Biol Chem 273: 8971-8974, 1998.

26. van Zon A, Mossink MH, Schoester M, Scheffer GL, Scheper RJ, Sonneveld $\mathrm{P}$ and Wiemer EA: Multiple human vault RNAs: expression and association with the vault complex. J Biol Chem 276: 37715-37721, 2001.

27. Kitazono M, Okumura H, Ikeda R, Sumizawa T, Furukawa $\mathrm{T}$, Nagayama S, Seto K, Aikou T and Akiyama S: Reversal of LRP-associated drug resistance in colon carcinoma SW-620 cells. Int J Cancer 91: 126-131, 2001

28. Patel J and Mitra AK: Strategies to overcome simultaneous P-glycoprotein mediated efflux and CYP3A4 mediated metabolism of drugs. Pharmacogenomics 2: 401-415, 2001

29. Siva AC, Raval-Fernandes S, Stephen AG, LaFemina MJ, Scheper RJ, Kickhoefer VA and Rome LH: Up-regulation of vaults may be necessary but not sufficient for multidrug resistance. Int J Cancer 92: 195-202, 2001.

30. Persson H, Kvist A, Vallon-Christersson J, Medstrand P, Borg A and Rovira C: The non-coding RNA of the multidrug resistance-linked vault particle encodes multiple regulatory small RNAs. Nat Cell Biol 11: 1268-1271, 2009.

31. Michaelis L and Menten ML: Die kinetik der invertinwirkung. Biochem Z 49: 333-369, 1913.

32. Livak KJ and Schmittgen TD: Analysis of relative gene expression data using real-time quantitative PCR and the 2(-Delta Delta C(T)) Method. Methods 25: 402-408, 2001. 